\title{
Multicenter retrospective analysis of patients with chronic lymphocytic leukemia in Korea
}

\begin{abstract}
Jun Ho Yi ${ }^{1}$, Gyeong-Won Lee ${ }^{2}$, Ji Hyun Lee ${ }^{3}$, Kwai Han Yoo ${ }^{4}$, Chul Won Jung ${ }^{5}$, Dae Sik Kim ${ }^{6}$, Jeong-Ok Lee ${ }^{7}$, Hyeon Seok Eom ${ }^{8}$, Ja Min Byun ${ }^{9}$, Youngil Koh ${ }^{9}$, Sung Soo Yoon ${ }^{9}$, Jin Seok Kim ${ }^{10}$, Jee Hyun Kong ${ }^{11}$, Ho-Young Yhim ${ }^{12}$, Deok-Hwan Yang ${ }^{13}$, Dok Hyun Yoon ${ }^{14}$, Do Hyoung Lim ${ }^{15}$, Won-Sik Lee ${ }^{16}$, Ho-Jin Shin ${ }^{17}$

${ }^{1}$ Division of Hematology-Oncology, Department of Medicine, Chung-Ang University Hospital, Seoul, ${ }^{2}$ Division of

Hematology-Oncology, Department of Internal Medicine, Institute of Health Science, Gyeongsang National University Hospital, Gyeongsang National University College of Medicine, Jinju, ${ }^{3}$ Department of Internal Medicine, Dong-A University College of Medicine, Busan, ${ }^{4}$ Division of Hematology-Oncology, Gachon University College of Medicine, Incheon, ${ }^{5}$ Department of Medicine, Samsung Medical Center, Sungkyunkwan University School of Medicine, ${ }^{6}$ Department of Internal Medicine, Korea University Guro Hospital, Seoul, ${ }^{7}$ Division of Hematology-Oncology, Seoul National University Bundang Hospital, Seongnam, ${ }^{8}$ National Cancer Center, Goyang, ${ }^{9}$ Division of Hematology-Oncology, Seoul National University Hospital, ${ }^{10}$ Division of Hematology, Department of Internal Medicine, Severance Hospital, Yonsei University College of Medicine, Seoul, ${ }^{11}$ Division of Hematology-Oncology, Department of Internal Medicine, Yonsei University Wonju College of Medicine, Wonju, ${ }^{12}$ Department of Internal Medicine, Jeonbuk National University Medical School, Jeonju, ${ }^{13}$ Department of Hematology-Oncology, Chonnam National University Hwasun Hospital, Gwangju, ${ }^{14}$ Department of Oncology, Asan Medical Center, University of Ulsan College of Medicine, Seoul, ${ }^{15}$ Division of Hematology-Oncology, Department of Internal Medicine, Dankook University College of Medicine, Cheonan, ${ }^{16}$ Inje University Busan Paik Hospital, ${ }^{17}$ Division of Hematology-Oncology, Department of Internal Medicine, School of Medicine, Medical Research Institute, Pusan National University Hospital, Busan, Korea
\end{abstract}

p-ISSN 2287-979X / e-ISSN 2288-0011 https://doi.org/10.5045/br.2021.2021102 Blood Res 2021;56:243-251.

Received on May 21, 2021

Revised on July 12, 2021

Accepted on August 25, 2021

\section{Correspondence to}

Ho-Jin Shin, M.D., Ph.D.

Division of Hematology-Oncology,

Department of Internal Medicine, School of Medicine, Medical Research Institute, Pusan National University Hospital, 179 Gudeok-ro, Seo-gu, Busan 49241, Korea E-mail: hojinja@hanmail.net

(C) 2021 Korean Society of Hematology

\section{Background}

Chronic lymphocytic leukemia (CLL) is the most common type of adult leukemia in Western countries but is rare in the East Asian countries. Due to its rarity and the lack of feasible novel agents and laboratory prognostic tools, there are limited data on the clinical outcomes of this disease in Asia. To clarify the current treatment status, we performed a multicenter retrospective analysis of patients with CLL in Korea.

\section{Methods}

The medical records of 192 eligible patients between 2008 and 2019 were reviewed for clinical characteristics, treatment courses, and outcomes. The first-line treatment regimens of the patients included in this analysis were as follows: fludarabine/cyclophosphamide/rituximab (FCR) ( $\mathrm{N}=117,52.7 \%)$, obinutuzumab plus chlorambucil (GC) $(\mathrm{N}=30,13.5 \%)$, and chlorambucil monotherapy $(\mathrm{N}=24,10.8 \%)$.

\section{Results}

The median progression-free survival (PFS) was 55.6 months, and the average 2-year PFS rate was $80.3 \%$. PFS was not significantly different between the patients receiving FCR and those receiving GC; however, chlorambucil treatment was associated with significantly inferior PFS $(P<0.001)$. The median overall survival was 136.3 months, and the average 5 - and 10 -year OS rates were $82.0 \%$ and $57.4 \%$, respectively.

\section{Conclusion}

This is one of the largest studies involving Korean patients with CLL. Although the patients had been treated with less favored treatment regimens, the outcomes were not different from those reported in Western studies.

Key Words Chronic lymphocytic leukemia, Outcomes, Asia 


\section{INTRODUCTION}

Chronic lymphocytic leukemia (CLL) is a neoplasm composed of monomorphic small mature $\mathrm{B}$ cells that co-express CD5 and CD23. In Western countries, it is the most common type of adult leukemia [1]. In 2019, it was estimated that 20,720 people would be diagnosed with CLL in the United States and 3,930 would die from the disease [2]. The clinical course of the disease is extremely heterogeneous, as some patients with favorable factors may not require treatment, whereas those with unfavorable risk factors may need treatment immediately $[3,4]$. Although the overall survival (OS) of CLL patients has improved over the past several decades owing to the introduction of novel agents $[5,6]$, these agents are costly and not readily available in real-world settings.

The incidence of CLL in Asia is approximately 20-fold lower than that in Western countries [7]. Moreover, people of Asian descent in Western countries present a lower incidence of CLL, suggesting that genetic factors play a bigger role than environmental factors in the development of CLL [8, 9]. In Korea, there were 157 cases of CLL in 2012, with an age-standardized rate of 0.2 [10]. Although its incidence is slowly increasing, CLL is considered a very rare disease that does not attract the attention of physicians. Consequently, the prognosis of CLL patients in Asia seems to be less favorable, probably due to the more aggressive biological features in Asian patients or the limited attention in healthcare settings $[11,12]$. Furthermore, the lack of feasible novel agents and laboratory prognostic tools may contribute to poor prognosis. We performed a nationwide, multicenter, retrospective analysis in Korea to clarify the current treatment status of CLL patients.

\section{MATERIALS AND METHODS}

\section{Patients}

This retrospective study included CLL patients treated with alkylating agents, purine analogs, or anti-CD20 monoclonal antibodies at 18 tertiary institutions in Korea. Their medical records were reviewed for clinical characteristics, treatment courses, and outcomes.

Patients who were treated for CLL between 2008 and 2019 were included in the analysis. The inclusion criteria were as follows: 1) patients with a primary diagnosis of CLL according to the International Workshop on Chronic Lymphocytic Leukemia (iwCLL) criteria [13]; 2) patients who had received therapy for CLL; and 3) availability of reliable medical records for the analysis. The patients' medical records regarding age, sex, Rai staging, Binet staging, Eastern Cooperative Oncology Group performance status (ECOG-PS), treatment regimens, cytogenetics, and laboratory findings including complete blood count and creatinine clearance $(\mathrm{CCr})$ rate were examined.

\section{Analysis}

Progression-free survival (PFS) was calculated from the date of treatment initiation for CLL to the date of progression or death, while OS was calculated from the date of treatment initiation to the date of death by any cause. Both PFS and OS were calculated using the Kaplan-Meier method, and subgroup comparisons were performed using the log-rank test. Responses were assessed using the iwCLL criteria [13]

Table 1. Clinical and molecular features during first-line treatment $(\mathrm{N}=192)$

\begin{tabular}{|c|c|}
\hline & $\mathrm{N}(\%)$ \\
\hline Age (median, range) & $63(34-87)$ \\
\hline$\leq 65 \mathrm{yr}$ & $117(60.9)$ \\
\hline$\geq 66 \mathrm{yr}$ & $75(39.1)$ \\
\hline \multicolumn{2}{|l|}{ Sex } \\
\hline Male & $115(59.9)$ \\
\hline Female & $77(40.1)$ \\
\hline \multicolumn{2}{|l|}{ ECOG-PS } \\
\hline $0-1$ & $179(93.2)$ \\
\hline $2-4$ & $13(6.8)$ \\
\hline \multicolumn{2}{|l|}{ Rai stage } \\
\hline 0 & $1(0.5)$ \\
\hline I & $46(24.0)$ \\
\hline II & $46(24.0)$ \\
\hline III & $50(26.0)$ \\
\hline IV & $49(25.5)$ \\
\hline \multicolumn{2}{|l|}{ Binet stage } \\
\hline A & $22(11.5)$ \\
\hline B & 76 (39.6) \\
\hline $\mathrm{C}$ & $94(49.0)$ \\
\hline \multicolumn{2}{|l|}{ Creatinine clearance $(\mathrm{N}=174)$} \\
\hline$\sim 69 \mathrm{~mL} / \mathrm{min}$ & $42(24.1)$ \\
\hline $70 \mathrm{~mL} / \mathrm{min}$ & $132(75.9)$ \\
\hline \multicolumn{2}{|l|}{ Largest tumor diameter } \\
\hline$\leq 5 \mathrm{~cm}$ & $180(93.7)$ \\
\hline$>5 \mathrm{~cm}$ & $12(6.3)$ \\
\hline \multicolumn{2}{|l|}{ FISH for del(11q) $(\mathrm{N}=51)$} \\
\hline Normal & $43(84.3)$ \\
\hline Abnormal & $8(15.7)$ \\
\hline \multicolumn{2}{|l|}{ FISH for del(13q) $(N=42)$} \\
\hline Normal & $24(57.1)$ \\
\hline Abnormal & $18(42.9)$ \\
\hline \multicolumn{2}{|l|}{ FISH for del(17p) $(N=46)$} \\
\hline Normal & $39(84.8)$ \\
\hline Abnormal & $7(15.2)$ \\
\hline \multicolumn{2}{|l|}{ TP53 mutation analysis $(\mathrm{N}=66)$} \\
\hline Mutated & $10(15.2)$ \\
\hline Wild-type & $56(84.8)$ \\
\hline \multicolumn{2}{|c|}{ FISH for del(17p) \& TP53 mutation analysis $(\mathrm{N}=26)$} \\
\hline Wild-type & $21(80.8)$ \\
\hline $\operatorname{Del}(17 p)$ & $1(3.8)$ \\
\hline Mutated TP53 & $1(3.8)$ \\
\hline $\operatorname{Del}(17 p) \&$ mutated TP53 & $3(11.5)$ \\
\hline \multicolumn{2}{|l|}{ IgHV mutation analysis $(\mathrm{N}=10)$} \\
\hline Mutated & $0(0.0)$ \\
\hline Wild-type & $10(100.0)$ \\
\hline
\end{tabular}

Abbreviations: del, deletion; ECOG-PS, Eastern Cooperative Oncology Group performance status; FISH, fluorescence in situ hybridization; $I g H V$, immunoglobulin heavy chain gene. 
and compared using Pearson's $\lambda^{2}$ tests. For all statistical analyses, $P$-values $<0.05$ were considered statistically significant; the analyses were performed using IBM Statistical Product and Service Solutions Statistics for Windows, version 21.0 (IBM Corp, Armonk, NY, USA).

All procedures performed in this study were in accordance with the ethical standards of the institutional committee. The Institutional Review Boards of the respective centers approved the study protocol.

\section{RESULTS}

\section{Patient characteristics and features at diagnosis}

From 2008 to 2019, 192 patients were eligible for the analysis, and all patients were Asian. The median age at diagnosis was 62 years (range, 34-86), and 115 patients (59.9\%) were male. The distribution of the patients according to the Rai stages at diagnosis was as follows: 0, 14 (7.3\%); I, 55 (28.6\%); II, 49 (25.5\%); III, 36 (18.8\%); and IV, 38 (19.8\%). The distribution of the patients according to the Binet stages at diagnosis was as follows: A, 45 (23.4\%); B,
80 (41.7\%); and C, 67 (34.9\%).

Treatment was initiated after a median observation period of 1.1 months (range, 0.0-135.8), when the patients' median age was 63 years (range, 34-87). The distribution of the patients according to the Rai stages during treatment was as follows: 0, 1 (0.5\%); I, 46 (24.0\%); II, 46 (24.0\%); III, 50 (26.0\%); and IV, 49 (25.5\%). The distribution of the patients according to the Binet stages during treatment was as follows: A, 22 (11.5\%); B, 76 (39.6\%); and C, 94 (49.0\%). The ECOG-PS of most patients was 0-1 ( $\mathrm{N}=179,93.2 \%)$, and the median $\mathrm{CCr}$ rate was $81.9 \mathrm{~mL} / \mathrm{min}$. Bulky disease characterized by lymph node(s) $>5 \mathrm{~cm}$ in dimension was observed in 12 patients (6.3\%), and B symptoms were present in 52 patients (27.1\%). Molecular analyses were not performed for all cases. Fluorescence in situ hybridization (FISH) for 17p deletion (del) was performed in 46 cases (23.9\%), of which 7 (15.2\%) tested positive. TP53 mutation analysis was performed in 66 cases (34.4\%), of which 10 (15.2\%) tested positive. Both FISH for del(17p) and TP53 mutation analysis were performed in only 26 cases, and 21 cases tested negative for both. Immunoglobulin heavy chain (IgHV) gene mutation analysis was performed in only 10 cases, and no
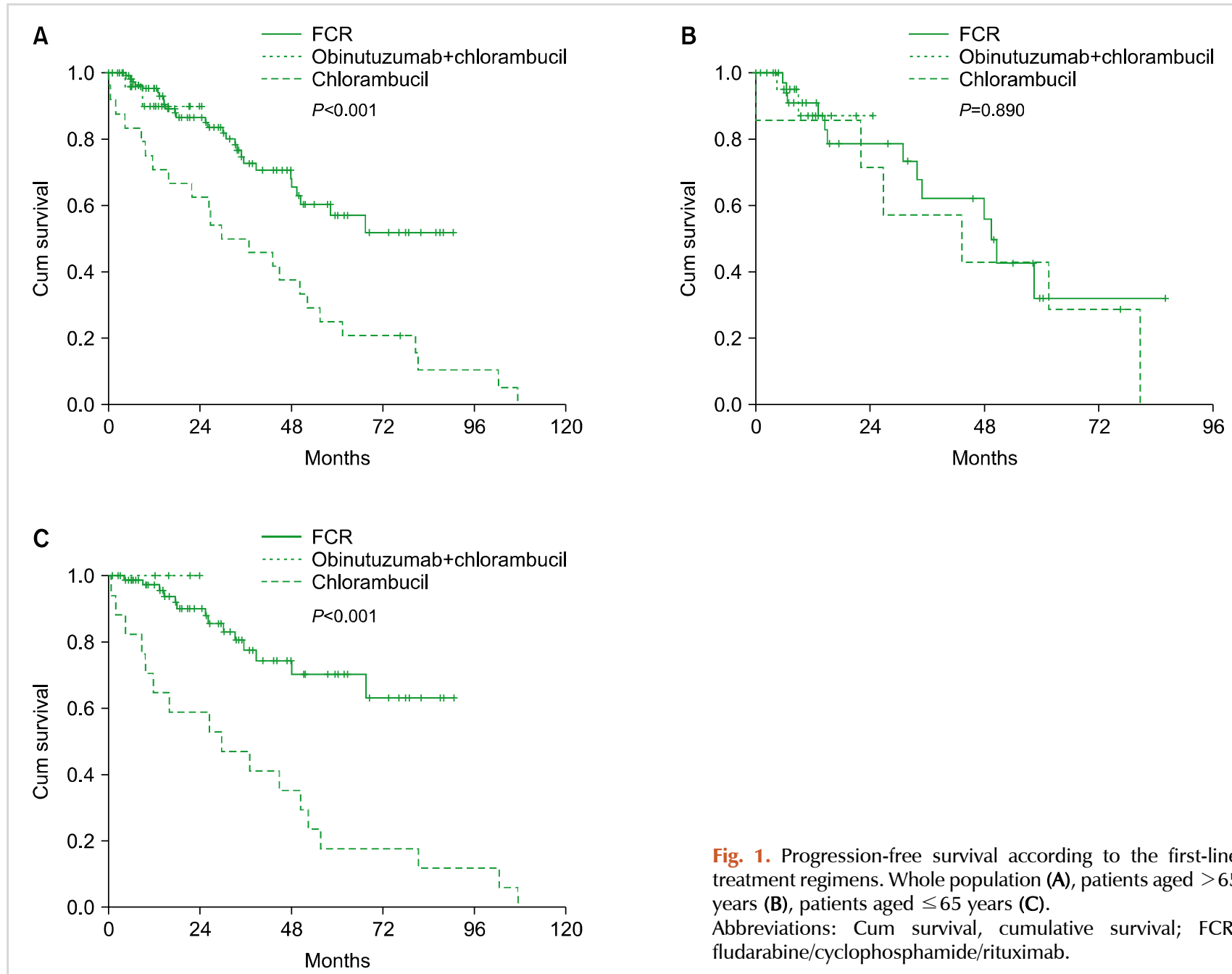

Fig. 1. Progression-free survival according to the first-line treatment regimens. Whole population (A), patients aged $>65$ years (B), patients aged $\leq 65$ years $(C)$.

Abbreviations: Cum survival, cumulative survival; FCR, fludarabine/cyclophosphamide/rituximab. 
mutation was detected in them. The clinical and molecular features of the patients are summarized in Table 1 .

\section{First-line treatment and outcomes}

The fludarabine, cyclophosphamide, and rituximab (FCR) regimen was the most commonly used $(\mathrm{N}=117,60.9 \%)$, followed by obinutuzumab plus chlorambucil (GC) ( $\mathrm{N}=30$, $15.6 \%)$. Other regimens included chlorambucil alone ( $\mathrm{N}=24$, $12.5 \%)$, fludarabine and cyclophosphamide $(\mathrm{N}=12)$, other fludarabine-based combinations $(\mathrm{N}=4)$, rituximab + bendamustine $(\mathrm{N}=4)$, and ibrutinib $(\mathrm{N}=1)$. The baseline characteristics of the patients according to the three major regimens (FCR, GC, and chlorambucil alone) are provided in Supplementary Table 1. The median PFS was 55.6 months [95\% confidence interval (CI), 40.4-70.8], and the mean \pm standard deviation (SD) 2-year PFS rate was $80.3 \pm 3.4 \%$. Complete response was achieved in 105 patients $(54.7 \%)$ and partial response in 51 patients $(26.6 \%)$, resulting in an overall response rate of $81.3 \%$. There was no difference in the PFS between patients treated with FCR and those with GC $(P=0.279)$. However, patients treated with chlorambucil alone showed a significantly shorter PFS than those treated with FCR or with GC $(P=0.001)$. The mean \pm SD 2-year PFS rates of the patients treated with FCR, GC, and chlorambucil were $86.6 \pm 3.7 \%, 89.8 \pm 6.9 \%$, and $62.5 \pm 9.9 \%$, respectively (Fig. 1A).

None of the age cut-off values, including 60,65 , and 70 years, could discriminate PFS (data not shown). However, analysis of the PFS for each regimen according to age showed interesting results. Among patients aged $\geq 66$ years, there

Table 2. Progression-free survival following first-line treatment according to clinical and molecular features.

\begin{tabular}{|c|c|c|c|}
\hline & $N$ & Median PFS $(95 \% \mathrm{Cl})$ & $P$ \\
\hline $\operatorname{Del}(17 p)$ & & & 0.043 \\
\hline Present & 7 & $9.0(5.1-12.9)$ & \\
\hline Absent & 39 & $52.3(32.5-72.1)$ & \\
\hline TP53 mutation & & & 0.005 \\
\hline Mutated & 10 & $12.8(3.9-21.5)$ & \\
\hline Wild type & 56 & $44.9(29.8-60.0)$ & \\
\hline $\operatorname{Del}(13 q)$ & & & 0.133 \\
\hline Present & 18 & $49.5(45.9-53.1)$ & \\
\hline Absent & 24 & $30.1(11.7-48.5)$ & \\
\hline $\operatorname{Del}(11 q)$ & & & 0.053 \\
\hline Present & 8 & $29.8(2.0-57.6)$ & \\
\hline Absent & 43 & $49.5(31.2-67.8)$ & \\
\hline Age & & & 0.258 \\
\hline$\leq 65 \mathrm{yr}$ & 117 & $67.5(37.6-97.4)$ & \\
\hline$\geq 66 \mathrm{yr}$ & 75 & $50.5(34.8-66.2)$ & \\
\hline Binet stage & & & 0.156 \\
\hline A & 22 & $47.9(14.7-81.1)$ & \\
\hline B & 76 & $55.6(28.1-83.1)$ & \\
\hline $\mathrm{C}$ & 94 & $58.4(31.1-85.7)$ & \\
\hline Rai stage & & & 0.876 \\
\hline 0 & 1 & NA & \\
\hline I & 46 & $61.5(46.6-76.4)$ & \\
\hline II & 46 & $47.9(32.3-63.5)$ & \\
\hline III & 50 & Not reached & \\
\hline IV & 49 & $58.4(39.6-77.2)$ & \\
\hline Treatment regimen & & & 0.001 \\
\hline FCR & 117 & Not reached & \\
\hline Obinutuzumab $+\mathrm{Cbl}$ & 30 & Not reached & \\
\hline $\mathrm{Cbl}$ & 24 & $29.8(9.8-49.8)$ & \\
\hline Patients aged $\leq 65 \mathrm{yr}$ & & & $<0.001$ \\
\hline FCR & 82 & Not reached & \\
\hline Obinutuzumab $+\mathrm{Cbl}$ & 5 & $21.4(\mathrm{NE})$ & \\
\hline $\mathrm{Cbl}$ & 17 & $29.8(1.3-58.3)$ & \\
\hline Patients aged $\geq 66 \mathrm{yr}$ & & & 0.890 \\
\hline FCR & 35 & $49.5(45.1-53.9)$ & \\
\hline Obinutuzumab $+\mathrm{Cbl}$ & 25 & Not reached & \\
\hline $\mathrm{Cbl}$ & 7 & $43.2(0.9-85.5)$ & \\
\hline
\end{tabular}

Abbreviations: Cbl, chlorambucil; Cl, confidence interval; Del, deletion; FCR, fludarabine/cyclophosphamide/rituximab; NA, not applicable; PFS, progression-free survival. 
was no significant difference in the mean \pm SD 2-year PFS rates between the three regimens: $78.6 \pm 7.9 \%$ for the FCR arm, $87.1 \pm 8.8 \%$ for the GC arm, and $71.4 \pm 17.1 \%$ for the chlorambucil arm $(P=0.890)$ (Fig. 1B). In contrast, a marked difference was observed in the PFS among the patients aged $\leq 65$ years (Fig. 1C). In this age group, the mean \pm SD 2-year PFS rate of the patients treated with FCR was $90.1 \pm 3.9 \%$, which was superior to that of patients treated with GC $(50.0 \pm 35.4 \%)$ or with chlorambucil $(58.8 \pm 11.9 \%)(P<0.001)$ (Table 2).

\section{Second-line treatment and outcomes}

In total, 57 patients received second-line treatment at a median age of 64 years (range, 44-86). Ibrutinib was the most frequently used second-line treatment regimen $(\mathrm{N}=24$, $42.1 \%)$, followed by FCR ( $\mathrm{N}=16,28.1 \%)$. Other regimens included fludarabine-based combinations ( $\mathrm{N}=6,10.5 \%)$, chlorambucil alone $(\mathrm{N}=3,5.2 \%)$, and bendamustine with or without rituximab $(\mathrm{N}=3,5.2 \%)$. The median PFS was
23.6 months (95\% CI, 5.0-42.2), and neither treatment regimen nor age could discriminate PFS (data not shown). The median PFS of patients who received ibrutinib was 23.6 months (95\% CI, 9.7-37.5), whereas that of patients who received FCR was 32.8 months (95\% CI, 0.0-68.4). As only 10 patients were examined for $\operatorname{del}(17 \mathrm{p})$ or TP53 mutation, and only one patient tested positive for $\operatorname{del}(17 p)$, their effects on PFS were not analyzed.

\section{OS and prognostic factors}

With a median follow-up duration of 80.3 months $(95 \%$ CI, 73.42-87.18), the median OS was 136.3 months (95\% CI, 100.1-172.5), and the mean \pm SD 5- and 10-year OS rates were $82.0 \pm 3.3 \%$ and $57.4 \pm 10.7 \%$, respectively (Fig. 2A). Several clinical and cytogenetic features discriminated the OS. An age cut-off of 65 years could not discriminate OS; however, patients aged <60 years (median, $154.0 \mathrm{mo}$; 95\% CI, 99.4-208.6) showed a significantly prolonged OS than that of patients aged $\geq 60$ years (median, 104.2 mo; $95 \%$

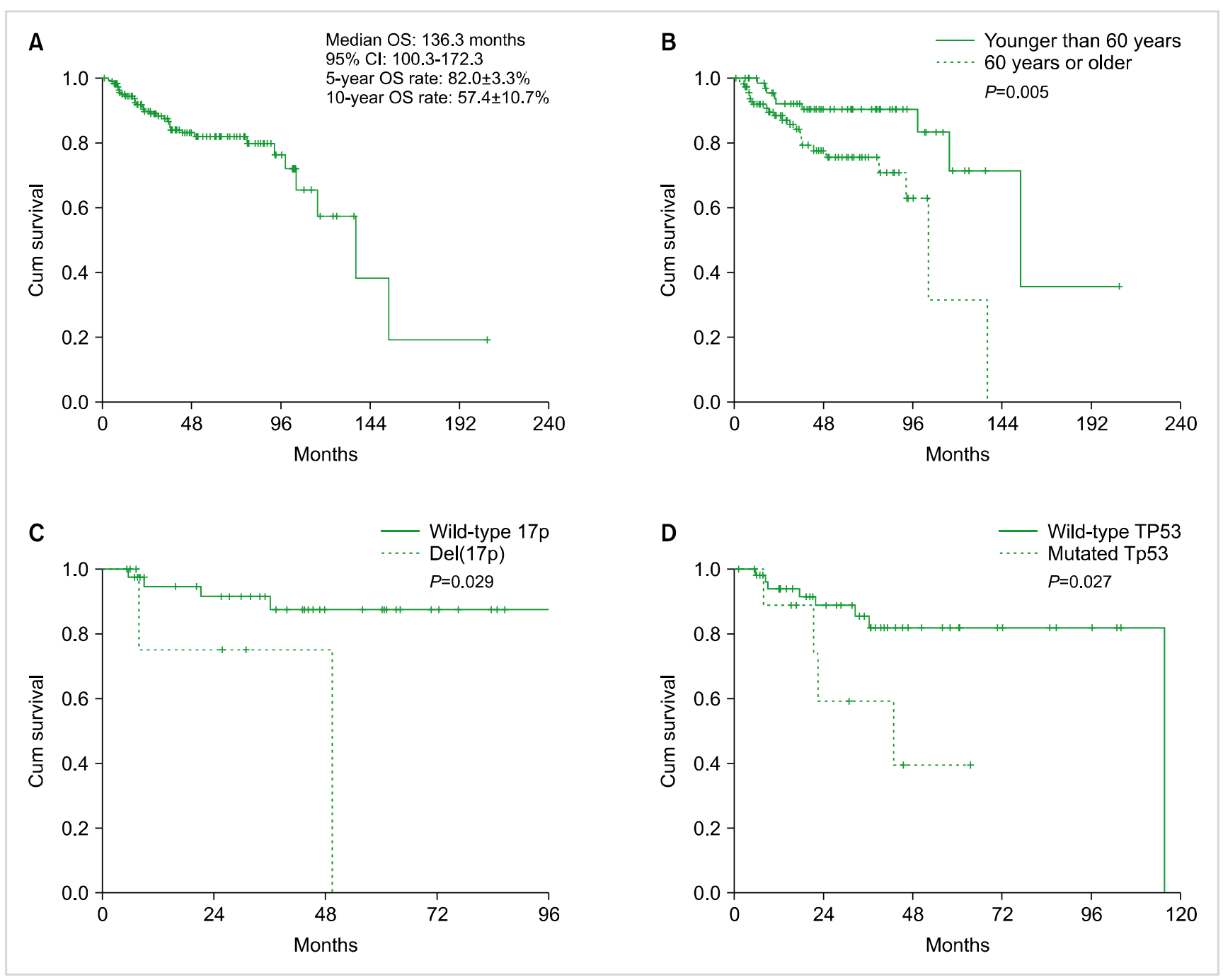

Fig. 2. Overall survival. Survival in the whole population (A), survival according to age (B), survival according to 17p deletion status (C) survival according to TP53 mutation status (D).

Abbreviations: $\mathrm{Cl}$, confidence interval; Cum survival, cumulative survival; OS, overall survival. 
CI, 87.6-120.8; $P=0.005$ ) (Fig. 2B). Neither the Rai stage nor the Binet stage could discriminate OS (data not shown). No significant difference was observed according to the first-line treatment regimen (mean \pm SD 5-year OS rate: FCR arm, 79.7 $\pm 4.5 \%$; GC arm, $73.6 \pm 12.4 \%$; chlorambucil arm, 91.7 $\pm 5.6 \% ; P=0.549$ ).

Although cytogenetic analyses were performed only in some cases, several abnormalities were found to affect the OS. Patients with del(17p) had worse OS rates than those with wild-type 17p (median OS, 49.5 mo vs. not reached; $P=0.029$ ) (Fig. 2C). Additionally, patients with TP53 mutation had worse OS than that of patients with wild-type TP53 (median OS, 42.8 mo vs. $115.8 \mathrm{mo}$; $P=0.027$ ) (Fig. 2D). There were no statistically significant differences in other cytogenetic abnormalities, including $\operatorname{del}(11 q)$ and $\operatorname{del}(13 q)$.

\section{DISCUSSION}

In this study, we analyzed 192 patients with CLL who were treated with alkylating agents, purine analogs, or anti-CD20 monoclonal antibodies. Treatment was initiated after a median observation period of 1.1 months (range, 0.0135.8), and the median PFS and OS were 55.6 months ( $95 \%$ CI, 40.4-70.8) and 136.3 months (95\% CI, 100.1-172.5), respectively. Age $\geq 60$ years, $\operatorname{del}(17 \mathrm{p})$, and TP53 mutations adversely affected OS.

CLL is a heterogeneous disease with highly variable initial presentation, necessitating a wide range of initial management strategies including watchful waiting, chemoimmunotherapy, Bruton's tyrosine kinase (BTK) inhibitors treatment, and B-cell lymphoma-2 (BCL-2) inhibitors treatment. As the outcomes of these treatments depend on several prognostic factors, it is crucial to evaluate the clinical and molecular markers comprehensively to determine the treatment strategies. However, although CLL is the most common type of adult leukemia in Western countries, its incidence in Asia is extremely low (5\%) as compared to that in Western countries $[14,15]$. This could be largely attributed to the genetic discordance between Caucasians and Asians [16, 17]. Consequently, the natural course and prognostic factors of CLL that are well defined for Caucasian patients $[3,4,18]$ are poorly defined for Asian patients.

Some studies have reported that the clinical and molecular features of Asian patients with CLL are different from those of the Western patients, including age, cytogenetics, and prognosis $[9,19]$. Corroborating with these reports, the median age of our patients was 62 years (range, 34-86), which was much lower than that of the patients in Western studies $[20,21]$. Despite the younger age at diagnosis, it has been reported that the disease progresses more aggressively in Asians [12, 22]. In this study, the mean \pm SD 5- and 10-year OS rates were $82.0 \pm 3.3 \%$ and $57.4 \pm 10.7 \%$, respectively, which were not significantly inferior to those reported in Western studies. This could be partially explained by the similarity with the Western studies in the proportion of patients with advanced stages of CLL, such as Binet stage C or Rai stages III-IV [20, 21]. In terms of cytogenetics, it seems that the incidence of major cytogenetic abnormalities, including $\operatorname{del}(11 q)$, del(13q), and $\operatorname{del}(17 p)$, is similar between Caucasians and Asians [3]; however, IgHV mutation is more frequently found in Asians ( 70\%) [23, 24]. Although a limited number of patients in this study were examined for $\operatorname{del}(11 q)$ (15.7\%), del(13q) (42.9\%), and $\operatorname{del}(17 p)(15.2 \%)$, the proportion of cytogenetic abnormalities was similar to that in previous studies. The status of $\operatorname{IgHV}$ mutation, which is important in the management of CLL patients, was only assessed in 10 patients, and none of them had the mutation. This could be because tailored treatment according to the $\operatorname{IgHV}$ mutation status is not permitted in Korea.

CLL therapy has undergone considerable changes over the last few years, and combinations of BTK inhibitors, BCL-2 inhibitors, and anti-CD20 monoclonal antibodies are recommended as the first-line treatment [25]. In this study, most patients were treated with FCR or chlorambucil with or without obinutuzumab, which are considered less favorable therapies. The average 2-year PFS rate reported for the FCR arm and GC arm was significantly higher than that of the chlorambucil arm. However, among elderly patients $(\geq 66$ $\mathrm{yr}$ ), there was no significant difference between the treatment arms. This finding is inconsistent with that of the CLL-11 trial in which elderly patients benefited from the combination treatment of obinutuzumab and chlorambucil [26]. One explanation for this is that $\operatorname{del}(17 \mathrm{p})$ was more common in patients treated with GC than in those treated with chlorambucil alone (33\% vs. $0.0 \%)$. As the current analysis began only 2 years after the approval of the treatment combination, the relatively short follow-up duration may have contributed to the biased results. Another interesting finding is that the differences in the PFS among the first-line treatment regimens did not lead to differences in the OS. This was also observed among patients who had received chlorambucil alone; the median OS of these patients was 136.3 months (95\% CI, 98.7-173.9), which was similar to that of the other patients. Similarly, Chinese [27] and Indian [28] trials reported a median OS of over 100 months with first-line chlorambucil treatment, but the Western studies reported different findings. In the CLL-11 trial, the median OS for the chlorambucil arm was 58.5 months after long-term follow-up [29]. In the COMPLEMENT-1 trial, the median OS for the chlorambucil arm was 84.7 months [30]. Although baseline molecular features should be considered, these findings suggest that chlorambucil monotherapy may be a suitable first-line option for CLL patients, especially when resources are limited.

This study has several limitations. The data were retrospectively collected from multiple institutes. This could lead to inconsistent interpretations of the responses. Nevertheless, as we assessed both PFS and OS, the validity of the data may be regarded as acceptable, and it may reflect real-world clinical outcomes. Furthermore, the outcomes of the first-line and second-line treatment regimens were heterogeneous, 
hindering the identification of an optimal treatment method for Asian patients with CLL. Although the data were collected from tertiary centers, analysis of various molecular markers was not performed. The disease may receive less attention from physicians due to its extremely low incidence, limited tailored treatment strategies, and novel first-line therapies.

In conclusion, this study analyzed the clinical and molecular features and treatment outcomes of 192 CLL patients in this study, and it is one of the largest nationwide studies in Korea to date. Although the patients had been treated with less favored agents, the outcomes were not different from those observed in Western studies. To determine the role of ethnicity in this "orphan" disease in Asia, further studies using novel agents are warranted.

\section{Authors' Disclosures of Potential Conflicts of Interest}

No potential conflicts of interest relevant to this article were reported.

\section{REFERENCES}

1. Teras LR, DeSantis CE, Cerhan JR, Morton LM, Jemal A, Flowers CR. 2016 US lymphoid malignancy statistics by World Health Organization subtypes. CA Cancer J Clin 2016;66:443-59.

2. Siegel RL, Miller KD, Jemal A. Cancer statistics, 2019. CA Cancer J Clin 2019;69:7-34.

3. Döhner H, Stilgenbauer S, Benner A, et al. Genomic aberrations and survival in chronic lymphocytic leukemia. N Engl J Med 2000;343:1910-6.

4. International CLL-IPI working group. An international prognostic index for patients with chronic lymphocytic leukaemia (CLL-IPI): a meta-analysis of individual patient data. Lancet Oncol 2016;17:779-90.

5. Abrisqueta $P$, Pereira A, Rozman $C$, et al. Improving survival in patients with chronic lymphocytic leukemia (1980-2008): the Hospital Clinic of Barcelona experience. Blood 2009;114:2044-50.

6. Brenner H, Gondos A, Pulte D. Trends in long-term survival of patients with chronic lymphocytic leukemia from the 1980s to the early 21st century. Blood 2008;111:4916-21.

7. Chihara D, Ito H, Matsuda T, et al. Differences in incidence and trends of haematological malignancies in Japan and the United States. Br J Haematol 2014;164:536-45.

8. Mak V, Ip D, Mang O, et al. Preservation of lower incidence of chronic lymphocytic leukemia in Chinese residents in British Columbia: a 26-year survey from 1983 to 2008. Leuk Lymphoma 2014;55:824-7.

9. Yang SM, Li JY, Gale RP, Huang XJ. The mystery of chronic lymphocytic leukemia (CLL): why is it absent in Asians and what does this tell us about etiology, pathogenesis and biology? Blood Rev 2015;29:205-13.

10. Lee H, Park HJ, Park EH, et al. Nationwide statistical analysis of lymphoid malignancies in Korea. Cancer Res Treat 2018;50: 22-38.

11. Wu SJ, Chiang CJ, Lin CT, Tien HF, Lai MS. Improving but inferior survival in patients with chronic lymphocytic leukemia in taiwan: a population-based study, 1990-2004. PLoS One 2013;8:e62930.

12. Gunawardana C, Austen B, Powell JE, et al. South Asian chronic lymphocytic leukaemia patients have more rapid disease progression in comparison to White patients. Br J Haematol 2008;142:606-9.

13. Hallek M, Cheson BD, Catovsky D, et al. iwCLL guidelines for diagnosis, indications for treatment, response assessment, and supportive management of CLL. Blood 2018;131:2745-60.

14. Yang S, Gale RP, Shi H, et al. Is there an epidemic of chronic lymphocytic leukaemia (CLL) in China? Leuk Res 2018;73:16-20.

15. Kim JS, Liu Y, Ha KH, Qiu H, Rothwell LA, Kim HC. Increasing incidence of B-cell non-Hodgkin lymphoma and occurrence of second primary malignancies in South Korea: 10-year follow-up using the Korean National Health Information Database. Cancer Res Treat 2020;52:1262-72.

16. Kawamata N, Moreilhon C, Saitoh T, et al. Genetic differences between Asian and Caucasian chronic lymphocytic leukemia. Int J Oncol 2013;43:561-5.

17. Kim JA, Hwang B, Park SN, et al. Genomic profile of chronic lymphocytic leukemia in Korea identified by targeted sequencing. PLoS One 2016;11:e0167641.

18. Chai-Adisaksopha C, Brown JR. FCR achieves long-term durable remissions in patients with IGHV-mutated CLL. Blood 2017;130: 278-82.

19. Lan $\mathrm{Q}, \mathrm{Au}$ WY, Chanock S, et al. Genetic susceptibility for chronic lymphocytic leukemia among Chinese in Hong Kong. Eur J Haematol 2010;85:492-5.

20. Sylvan SE, Asklid A, Johansson H, et al. First-line therapy in chronic lymphocytic leukemia: a Swedish nation-wide real-world study on 1053 consecutive patients treated between 2007 and 2013. Haematologica 2019;104:797-804.

21. Mato A, Nabhan C, Kay NE, et al. Real-world clinical experience in the Connect ${ }^{\mathbb{R}}$ chronic lymphocytic leukaemia registry: a prospective cohort study of 1494 patients across 199 US centres. Br J Haematol 2016;175:892-903.

22. Jang MA, Yoo EH, Kim K, et al. Chronic lymphocytic leukemia in Korean patients: frequent atypical immunophenotype and relatively aggressive clinical behavior. Int J Hematol 2013;97:403-8.

23. Chen L, Zhang Y, Zheng W, et al. Distinctive IgVH gene segments usage and mutation status in Chinese patients with chronic lymphocytic leukemia. Leuk Res 2008;32:1491-8.

24. Tomomatsu J, Isobe $\mathrm{Y}$, Oshimi K, et al. Chronic lymphocytic leukemia in a Japanese population: varied immunophenotypic profile, distinctive usage of frequently mutated IGH gene, and indolent clinical behavior. Leuk Lymphoma 2010;51:2230-9.

25. Wierda WG, Byrd JC, Abramson JS, et al. Chronic lymphocytic leukemia/small lymphocytic lymphoma, version 4.2020, NCCN Clinical Practice Guidelines in Oncology. J Natl Compr Canc Netw 2020;18:185-217.

26. Goede V, Fischer K, Busch R, et al. Obinutuzumab plus chlorambucil in patients with CLL and coexisting conditions. N Engl J Med 2014;370:1101-10.

27. Chan TS, Lee YS, Del Giudice I, et al. Clinicopathological features and outcome of chronic lymphocytic leukaemia in Chinese patients. Oncotarget 2017;8:25455-68.

28. Tejaswi V, Lad DP, Jindal N, et al. Chronic lymphocytic leukemia: real-world data from India. JCO Glob Oncol 2020;6:866-72. 
29. Goede V, Fischer K, Bosch F, et al. Updated survival analysis from the CLL11 study: obinutuzumab versus rituximab in chemoimmunotherapy-treated patients with chronic lymphocytic leukemia. Blood (ASH Annual Meeting Abstracts) 2015;126(Suppl):1733.

30. Offner F, Robak T, Janssens A, et al. Long-term follow-up of previously untreated patients (pts) with chronic lymphocytic leukemia (CLL) treated with ofatumumab (OFA) and chlorambucil (CHL): final analysis of the phase 3 COMPLEMENT 1 trial. J Clin Oncol (ASCO Annual Meeting Abstracts) 2019;37(Suppl 15):7528-8. 